\title{
The Intracranial Aneurysm Gene THSD1 Connects Endosome Dynamics to Nascent Focal Adhesion Assembly
}

\author{
Yan-Ning Rui ${ }^{a} \quad$ Zhen Xu ${ }^{a} \quad$ Xiaoqian Fanga,b Miriam R. Menezes ${ }^{a} \quad$ Julien Balzeau ${ }^{a}$ \\ Airu Niu ${ }^{a}$ John P. Hagan ${ }^{a}$ Dong H. Kimª \\ aDepartment of Neurosurgery, McGovern Medical School, The University of Texas Health Science \\ Center at Houston, Houston, TX, U.S.A. ${ }^{b}$ Current address: Department of Biomedical Sciences, The \\ University of Texas Rio Grande Valley School of Medicine, McAllen, TX, USA
}

\section{Key Words}

Thsd1 • Focal adhesion • Endosome • Protein complex

\begin{abstract}
Background/Aims: We recently discovered that harmful variants in THSD1 (Thrombospondin type-1 domain-containing protein 1) likely cause intracranial aneurysm and subarachnoid hemorrhage in a subset of both familial and sporadic patients with supporting evidence from two vertebrate models. The current study seeks to elucidate how THSD1 and patientidentified variants function molecularly in focal adhesions. Methods: Co-immunostaining and co-immunoprecipitation were performed to define THSD1 subcellular localization and interacting partners. Transient expression of patient-identified THSD1 protein variants and siRNA-mediated loss-of-function THSD1 were used to interrogate gene function in focal adhesion and cell attachment to collagen I in comparison to controls. Results: THSD1 is a novel nascent adhesion protein that co-localizes with several known markers such as FAK, talin, and vinculin, but not with mature adhesion marker zyxin. Furthermore, THSD1 forms a multimeric protein complex with FAK/talin/vinculin, wherein THSD1 promotes talin binding to FAK but not to vinculin, a key step in nascent adhesion assembly. Accordingly, THSD1 promotes mature adhesion formation and cell attachment, while its rare variants identified in aneurysm patients show compromised ability. Interestingly, THSD1 also localizes at different stages of endosomes. Clathrin-mediated but not caveolae-mediated endocytosis pathway is involved in THSD1 intracellular trafficking, which positively regulates THSD1-induced focal adhesion assembly, in contrast to the traditional role of endosomes in termination of integrin signals. Conclusions: The data suggest that THSD1 functions at the interface between endosome dynamics and nascent focal adhesion assembly that is impaired by THSD1 rare variants identified from intracranial aneurysm patients.
\end{abstract}

Y. Rui and Z. Xu contributed equally to this work.

Yan-Ning Rui

and John P. Hagan
Department of Neurosurgery, McGovern Medical School,

The University of Texas Health Science Center at Houston, Houston, TX, (USA)

E-Mail Yanning.Rui@uth.tmc.edu, John.P.Hagan@uth.tmc.edu 


\section{Cellular Physiology Cell Physiol Biochem 2017;43:2200-2211

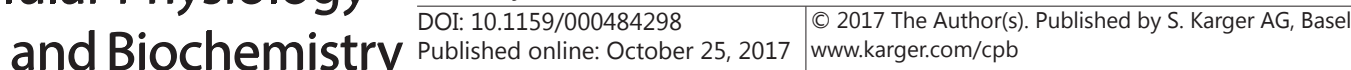

Rui et al.: Endosome-Mediated THSD1 Dynamics Regulate FA

\section{Introduction}

We recently identified a novel, nonsense variant in THSD1 that co-segregates with disease in an autosomal dominant manner in a large family [6 individuals with unruptured intracranial aneurysms (IA) and 3 individuals with subarachnoid hemorrhage (SAH)] using genome-wide linkage combined with whole exome sequencing analyses [1, 2]. Among 507 analyzed IA probands in our patient cohort, functionally significant THSD1 missense variants were discovered in $8 \mathrm{IA} / \mathrm{SAH}$ patients. Each identified variant adversely affected protein function. We showed that THSD1 is highly expressed in endothelial cells, and Thsd1 loss-of-function in both zebrafish and mice leads to premature death and cerebral hemorrhage that is localized to the subarachnoid space in mice. These phenotypes may be caused in part by defective focal adhesion, since reduced numbers of focal adhesion and levels of phosphorylated Focal Adhesion Kinase (FAK S397) were observed in THSD1deficient endothelial cells [2].

Focal adhesions (FA) are complex macromolecular structures that link extracellular matrix (ECM) interactions mediated by integrins to downstream signaling and the actin cytoskeleton [3]. FA are dynamic structures that form initially as nascent adhesions with a circular morphology upon ECM-integrin interaction that develop into mature adhesions with characteristic elongated morphology at the ventral and peripheral area of the cell. Scaffold proteins such as FAK, talin, and vinculin are recruited at nascent adhesions [4]. In particular, talin interacts with the cytoplasmic tail of integrin and activates integrin insideout signaling to facilitate its binding to ECM [5]. FAK directly interacts with talin and recruits it to nascent adhesions [6], supporting the important role of FAK-talin interaction in nascent adhesion formation.

In comparison to well-characterized mature adhesions [3], far less is known about how the assembly of nascent adhesions is regulated in general and more specifically, in cells that express THSD1 such as endothelial cells. Endocytic pathways may negatively regulate nascent adhesions since integrin is endocytosed in many cell types and routed to lysosome for degradation [7]. In contrast, endocytic pathways may promote nascent adhesions by delivering recycled integrin upon endosome-mediated redistribution. This idea is supported by a recent report that recycled integrin can maintain an active conformation when FAK localizes at recycling endosomes and subsequently promotes talin-mediated integrin activation [8]. However, it is still poorly understood how endocytic pathways regulate nascent adhesion formation.

Here, we report that the single-pass transmembrane protein THSD1 is localized to nascent adhesions and endosomes. THSD1 promotes FAK-talin interaction at nascent focal adhesions and accordingly increases the number of mature adhesions and enhances cellular attachment to ECM. Clathrin-mediated endocytosis appears to redistribute THSD1 that positively regulates THSD1-induced FA in part by providing recycled THSD1 to new nascent adhesion sites.

\section{Materials and Methods}

Plasmids:

pCMV5-THSD1-GFP and pCMV5-THSD1-FLAG were cloned by a newly-developed precision tagging technique [9]. Wild-type THSD1 or its variants including R450X, R460W, E466G G600E, P639L, T653I, and S775P were amplified by PCR from previous plasmids [2] and sub-cloned into pCMV5 vector. pCMV5, pCMV5-GFP or pCMV5-FLAG was used as negative control where appropriate.

Chemicals:

Sucrose (S7903), MDC (D4008), filipin III (F9765) and FLAG peptide (F3290) were from Sigma-Aldrich. All other chemicals such as paraformaldehyde gels was from Fisher Scientific. 


\section{Cellular Physiology Cell Physiol Biochem 2017;43:2200-2211 and Biochemistry \begin{tabular}{l|l} 
DOI: 10.1159/000484298 & $\begin{array}{l}\text { O 2017 The Author(s). Published by S. Karger AG, Basel } \\
\text { wwww.karger.com/cpb }\end{array}$
\end{tabular} and Biochemistry $\frac{\text { Published online. }}{\text { Rui et al.: Endosome-Mediated THSD1 Dynamics Regulate FA }}$}

SiRNAs:

Sequence of siRNAs against human THSD1 were 5'-tctgcaaaggaggagtgcatgctaa (\#1) and 5'-ttggtggtactctgtgactat (\#2). siTHSD1\#1 specificity was validated by full-length cDNA rescue experiments as described previously [2].

Cell culture and transfection:

HeLa and HEK293T cells were maintained in DMEM medium (Mediatech) containing 10\% fetal bovine serum as de. HUVEC cells were maintained in $\mathrm{EGM}^{\mathrm{TM}}$-2 Media (EGM-2 BulletKit, Lonza). Transfections of plasmid DNA or siRNAs were performed using lipofectamine 3000 (Invitrogen) or electroporation protocol (Neon ${ }^{\circledR}$ Transfection System, Invitrogen), respectively.

\section{Drug treatment:}

Hypertonic sucrose $(0.4 \mathrm{M})$, monodansylcadaverine $(100 \mu \mathrm{M})$ or filipin III $(5 \mu \mathrm{g} / \mathrm{ml})$ were added to HeLa or HEK293T cells expressing control or THSD1 for 1 hour prior to immunostaining.

\section{Immunoblotting:}

Transiently transfected HEK293T cells were lysed in a Triton X-100 lysis buffer (20 mM Tris-HCl, pH 7.4, 150 mM NaCl, 1 mM EDTA, 1 mM EGTA, 1\% Triton X-100, 2.5 mM sodium pyrophosphate, 1 mM $\beta$-glycerolphosphate, $1 \mathrm{mM}$ sodium orthovanadate, $1 \mu \mathrm{g} / \mathrm{ml}$ leupeptin) as previously described [10]. Total cell lysates were subjected to SDS-PAGE analysis using Bio-Rad mini transfer apparatus followed by blocking with 5\% non-fat milk. Primary antibodies and secondary antibodies were used usually at 1:1000 and 1:10000 dilutions, respectively before using Odyssey system to detect protein signals.

\section{Co-Immunoprecipitation}

Transiently transfected HEK293T cells were lysed in Triton X-100 lysis buffer as previously described [11]. THSD1-FLAG or talin was immunoprecipitated with anti-FLAG or anti-talin antibody plus protein A/G Plus agarose beads (Santa Cruz Biotechnology, sc-2003). Immunoprecipitates or total cell lysates (TCL) were subjected to immunoblotting. Two-step co-immunoprecipitation was described as previously reported [12, 13].

\section{Immunostaining:}

HeLa cells transfected with GFP or THSD1-GFP were expressed for 36-48 hours and fixed in 4\% paraformaldehyde for 10 mins. Fixed cells were blocked in 10\% Normal Goat Serum at room temperature for 1 hour and incubated with primary antibodies at $4{ }^{\circ} \mathrm{C}$ overnight. Alexa-594 or Alexa-488 conjugated secondary antibodies were used and images were captured by a Leica confocal microscope. Quantification of zyxin-positive puncta formation was similarly performed as previously described [2].

\section{Cell attachment:}

Cell attachment assay was performed based on a published protocol [14]. Briefly, HeLa cell were detached by non-enzymatic dissociation buffer (13151014, Thermosci Fisher) and reseeded onto 96-well polystyrene plate coated with $40 \mu \mathrm{g} / \mathrm{ml}$ collagen I (CC050, Millipore) for 30 mins. After the non-adherent cells were washed away by DMEM media, the remaining attached cells were quantified by crystal violetbased spectrophotometric method as previously described [2].

\section{Statistics analysis}

All in vitro experiments were performed in triplicates and repeated at least 3 times. Statistical analysis was performed with Microsoft Excel and p-values were calculated by the Student's t -test. Data are represented as mean \pm s.e.m. $\mathrm{P}<0.05$ is considered significant. 


\section{Cellular Physiology and Biochemistry

Fig. 1. THSD1 localizes at nascent but not mature adhesions. (A) 0.5ug THSD1-GFP was transfected into HeLa cells followed by co-immunostaining against both GFP and endogenous vinculin, highlighted by green and red, respectively. (B) Co-immunostaining of THSD1-GFP with mature FA marker zyxin in HeLa cells. (C-E) Co-immunostaining of THSD1GFP and vinculin (C), talin (D) or FAK (E). Arrows indicate co-localized signals. Enlarged pictures were shown on the lower panels. Scale bar: $2 \mu \mathrm{m}$. (F) Western blotting revealed the level of THSD1 by GFP antibody. GAPDH serves as loading control.

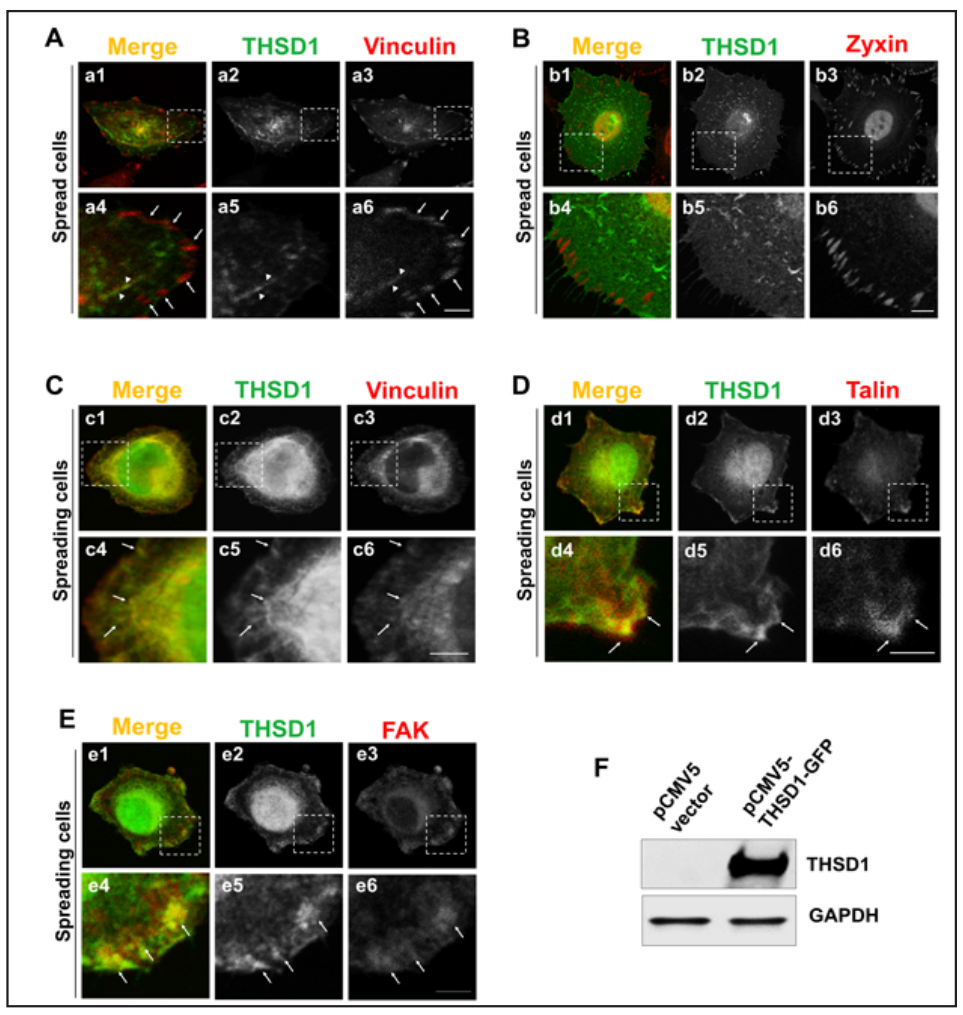

\section{Results}

THSD1 localizes to nascent FAs

We recently reported that THSD1 physically associates with talin [2], an adaptor protein that interacts with the cytoplasmic tail of integrin at FA sites. This result is consistent with the identification of THSD1 by protein mass spectrometry in talin immunopurification [15]. Therefore, we hypothesized that THSD1 may be a novel component of FA, a complex macromolecule structure that mediates integrin activation, downstream signaling, and actin cytoskeleton organization [3]. Since commercial antibodies qualified for immunostaining of endogenous THSD1 are not available, we generated a fusion construct that expresses GFP fusion at the C-terminal of THSD1 by our newly described precision tagging technique [9] and confirmed its expression by immunoblotting (Fig. 1F).

HeLa cells are widely used as a model for FA studies due to their strong attachment to the extracellular matrix. Accordingly, we performed co-immunostaining of transfected THSD1GFP and endogenous vinculin, a universal marker for FA. As shown in Fig. 1a2 and 1a5, THSD1 forms discrete puncta at both peripheral and ventral side of the cells. Surprisingly, the majority of these puncta do not colocalize with the ones highlighted by vinculin antibodies (see green and red signals in Fig. 1a4 and compare Fig. 1a5 and 1a6), especially those puncta with elongated morphology (white arrows in Fig. 1a4 and 1a6), which commonly indicate mature adhesions. To further confirm this observation, we examined another FA marker zyxin, which is only expressed in mature adhesions [16] and found almost no evidence of overlapping signals as shown in Fig. 1B (see green and red signals in the enlarged panel in Fig. 1b4, and compare Fig. $1 \mathrm{~b} 5$ with 1b6). Nonetheless, we did notice that THSD1 localizes at a few vinculin-positive small-sized dots (white arrowheads in Fig. 1a4-1a6) that are indicative of nascent FAs [4].

Nascent FAs are transient cytoskeletal structures linked to integrin complex, and become more prominent when spreading cells attach to the extracellular matrix [17]. Therefore, to enrich for nascent adhesions, we chose to stain the cells at $30 \mathrm{mins}$ after they were reseeded onto the slides. Nascent adhesions highlighted by vinculin were widely distributed at the 


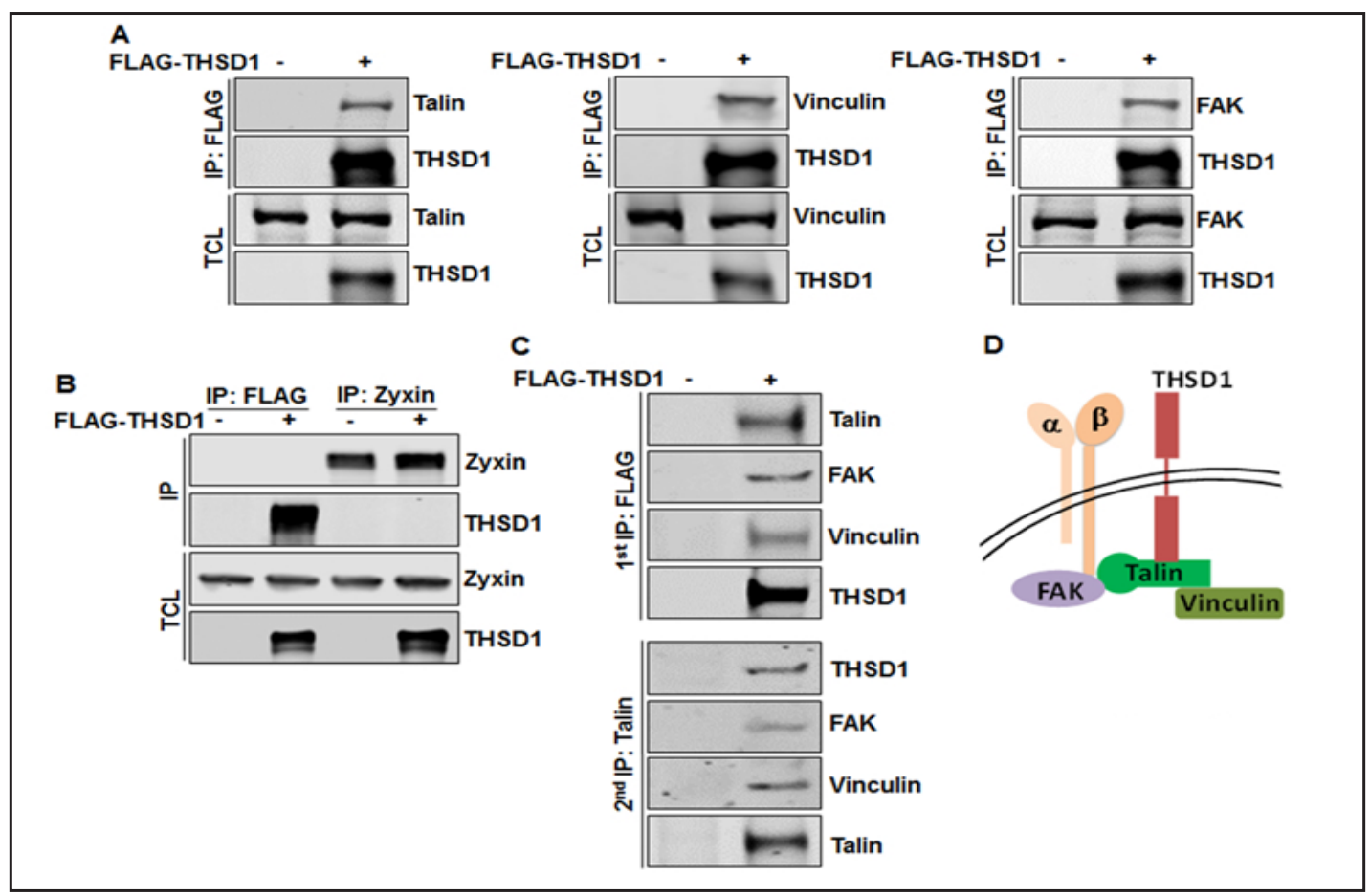

Fig. 2. THSD1 forms a multimeric protein complex with FAK-talin-vinculin. (A-B) HEK293T was transfected with 2 ug of either vector or THSD1-FLAG followed by M2 beads-based immunoprecipitation. THSD1interacting proteins were revealed by using antibodies against talin, vinculin, FAK, and zyxin. TCL is short for total cell lysate and IP for immunoprecipitation. (C) Two-step co-immunoprecipitation for THSD1 and FAKtalin-vinculin complex. In the first round IP, M2 beads were used to pull-down THSD1-associated complex FAK-talin-vinculin. THSD1 complex eluted by FLAG peptide was subjected to second round IP by anti-talin antibody. (D) Schematic of a multimeric protein complex comprising THSD1 and FAK-talin-vinculin together with $\alpha / \beta$ integrin.

protruding cell edges consistent with previous reports (Fig. 1c3 and 1c6) [6]. Interestingly, the majority of THSD1 puncta co-localize with vinculin-positive signals (white arrows in Fig. 1c4-c6). Since vinculin localizes at nascent adhesions by binding to talin that is recruited by FAK to integrin complex, we reasoned that THSD1 might also co-localize with talin and FAK. Indeed, strong co-localization signals were detected in the cells by antibodies against both THSD1 and talin or FAK (Fig. 1D-E), further supporting the notion that THSD1 is a novel component of nascent adhesions.

\section{Multimeric protein complex formation between THSD1 and FAK-talin-vinculin}

At nascent adhesions, FAK directly interacts with talin [6], which can bind to both vinculin and THSD1 [2]. To examine whether THSD1 can physically interact with FAK-talinvinculin complex, we performed a co-immunoprecipitation assay in HEK293T cells and confirmed the physical interaction between THSD1 and talin as previously observed in endothelial cells [2]. Endogenous talin was detected in the THSD1 immunoprecipitates but not in controls (comparing the second to the first lane in the left panel of Fig. 2A). Similarly, we found that both vinculin and FAK can be co-immunoprecipitated by antibodies against THSD1 (comparing the second to the first lane in the middle or the right panel of Fig. 2A). In contrast, we could not detect zyxin in the THSD1 immunoprecipitates (Fig. 2B), consistent with our previous observation that THSD1 is absent from the mature FAs.

To determine if THSD1 forms a multimeric protein complex with FAK-talin-vinculin rather than binding to them separately, we performed a two-step co-immunoprecipitation protocol as described previously [12]. First, we used anti-FLAG antibody to pull-down THSD1 
protein complex and consistently detected all other binding partners (upper panel in Fig. 2C). THSD1 complex was eluted by FLAG peptides and subjected to anti-talin antibody for the second-round immunoprecipitation. As shown in the lower panel in Fig. 2C, THSD1, FAK and vinculin were detected in the final talin immunoprecipitates, supporting that THSD1 localizes at nascent adhesions by physically interacting with FAK-talin-vinculin complex (model in Fig. 2D).

\section{THSD1 promotes FAK-talin interaction and FA number}

FAK-talin physical interaction is important for nascent adhesion formation [6]. To test how THSD1 may regulate its assembly, we carried out a series of binding affinity experiments according to our previous protocol [11]. In the absence of THSD1, we detected the physical interaction between endogenous FAK and Talin (the first panel in Fig. 3A). Interestingly, such interaction can be significantly enhanced when THSD1 was co-expressed in a dose-dependent manner (compare the amount of co-immunoprecipitated FAK in the second and the third lane with that in the first lane in Fig. 3A). In contrast, such binding affinity-promoting effect was abolished when cells were transfected with THSD1-R450X (Fig. 3B), a nonsense variant that encodes a truncated protein that removes almost the entire intracellular region and that co-segregates with disease in our previously reported CVM family (6 unruptured IA and 3

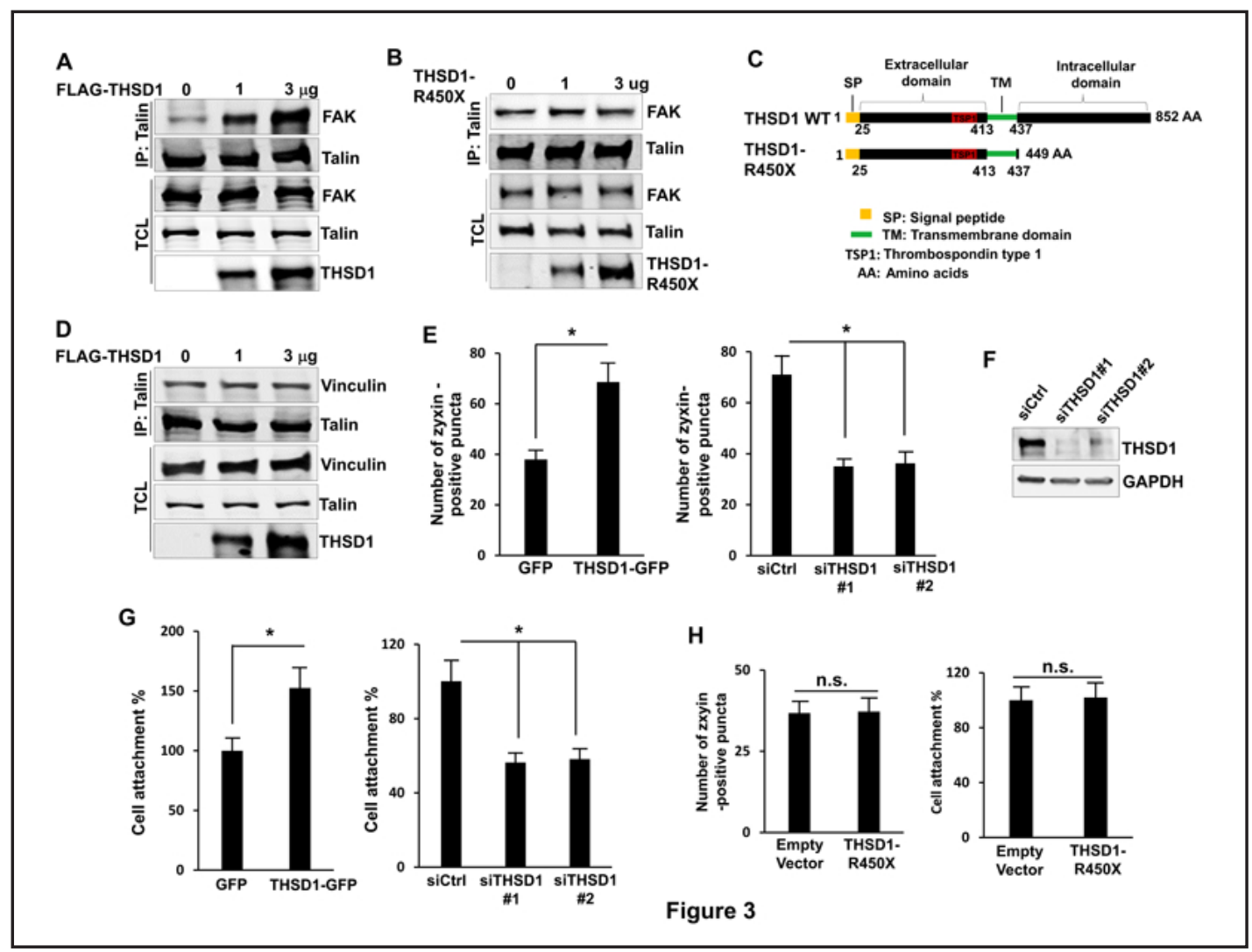

Fig. 3. THSD1 promotes FAK-talin interaction and FA. (A-D) 1 or $3 \mu \mathrm{g}$ of THSD1-FLAG was transfected into HEK293T cells and co-IPed FAK (A-B) or vinculin (D) was revealed by immunoblotting. Schematic of THSD1 R450X truncation variant is indicated in (C). (E-G) HeLa cells (left graph in E and G) were transfected with 2ug of control GFP or THSD1-GFP and HUVEC cells (right graph in E and G) were transfected with $10 \mathrm{nM}$ control siRNA or two independent THSD1 siRNAs. Zyxin antibody immunostaining (E) or cell attachment assay (G) were performed and quantification is described in Methods. THSD1 knockdown efficiency was revealed by immunoblotting using anti-THSD1 antibody (F). (H) HeLa cells with $2 \mathrm{ug}$ of empty vector or THSD1-R450X were immunostained against zyxin (left graph) and subjected to cell attachment assay (right graph) 
Fig. 4. THSD1 variants have a compromised ability to promote FAK-talin interaction. (A) Schematic of THSD1 missense variants (highlighted in red letters) (B-C) Empty vector or THSD1 including widetype (WT) and its variants (R460W, G00E, P639L, T653I, and $\mathrm{S775P}$ ) were transfected into HEK293T cells respectively. (B) Endogenous FAK was pull-down by anti-talin antibody in the presence of THSD1 variants. (C) THSD1 variants were co-immunoprecipitated by anti-talin antibody. (D-E) Mature focal adhesion number (D) and cell attachment (E) were assessed in the presence of different THSD1 variants.

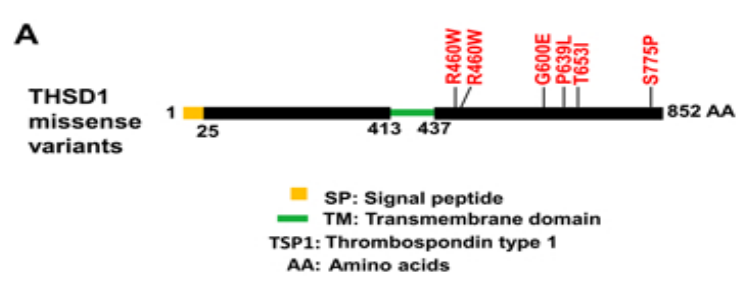

$\mathbf{B}$
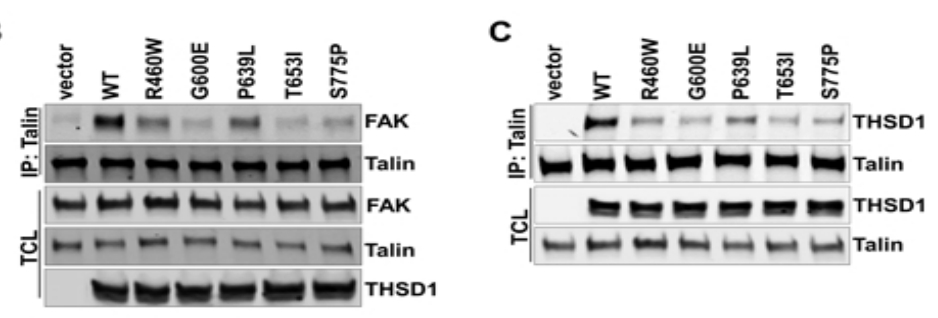

D

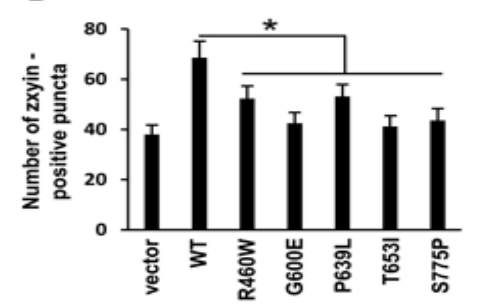

E

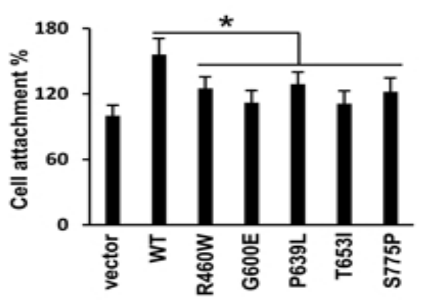

SAH) (Fig. 3C). Ectopic expression of THSD1 has little effect on the binding affinity between talin and vinculin (Fig. 3D).

Next, we tested whether THSD1 promotes the transition from nascent to mature focal adhesion. Ectopic expression of THSD1 in HeLa cells increases the number of mature adhesions labeled by zyxin (left graph in Fig. 3E), while knockdown of THSD1 in human umbilical vein endothelial cells (HUVEC) decreases it (right graph in Fig. 3E). The knockdown efficiency of THSD1 in HUVEC cells was confirmed by immunoblotting (Fig. 3F). These data support that THSD1 is a positive regulator of FA numbers.

Next, we examined how THSD1 regulates cell-extracellular matrix interaction where FA is critically involved. We performed cell attachment assays and found that THSD1 expression in HeLa cells promotes their binding to collagen I-coated plates, while knockdown of THSD1 in HUVEC cells decreases it (Fig. 3G), consistent with its positive role in FA numbers. In addition, both the number of mature adhesions and cell attachment was not affected by the expression of THSD1-R450X (Fig. 3H), suggesting that THSD1-promoted FAK-talin interaction is involved.

\section{THSD1 variants have compromised ability to promote FAK-talin interaction}

THSD1 is a single pass transmembrane protein with an $\mathrm{N}$-terminal extracellular region with a TSP1 domain and a C-terminal intracellular domain (Fig. 3C). In addition to the cosegregating, nonsense R450X variant identified in the CVM family characterized by autosomal dominant IA, several functionally significant missense THSD1 variants were discovered in our IA patient cohort (Fig. 4A) [2]. Therefore, we tested the behavior of six patient-identified THSD1 missense variants (R460W, E466G, G600E, P639L, T653I, and S775P) [2] in terms of promoting FAK-talin interaction and FA number. In HEK293T cells, the E466G protein variant was poorly expressed and/or unstable that led to normalization difficulty and was excluded from further experiments. All tested THSD1 variants showed compromised ability to promote FAK-talin interaction, in comparison with wild-type THSD1 (comparing lane

\section{KARGER}


Fig. 5. THSD1 localizes at different stages of endosomes. (A-D) 0.5ug of THSD1-GFP was transfected into HeLa cells. (A) EEA1, an early endosome marker. (B) CIMPR, a late endosome marker, (C) Rab4, a recycling endosome marker, and (D) LAMP1, a lysosome marker, were separately co-immunostained with THSD1-GFP. Scale bar: $2 \mu \mathrm{m}$.

3-7 to lane 2 in Fig. 4B). To check whether such effects are caused by the reduced binding of THSD1 to talin, we examined the physical interactions between talin and different THSD1 variants. Indeed, compared to wild-

A

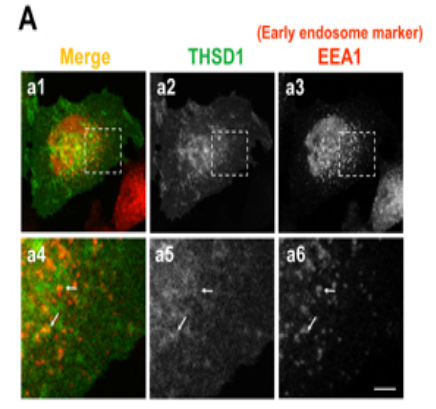

B

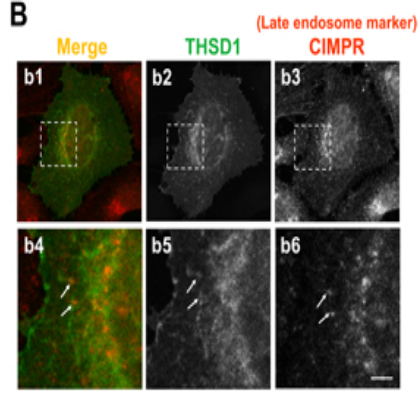

C

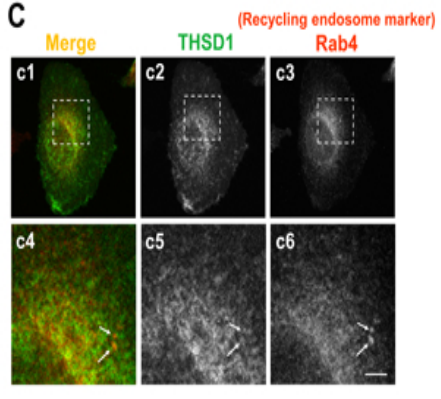

D

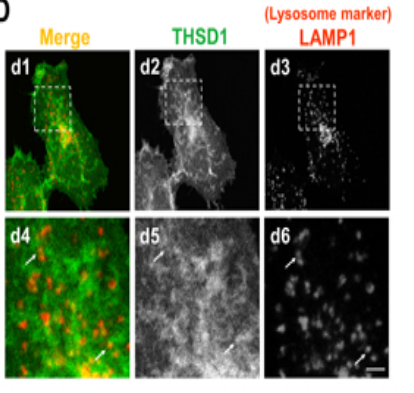
type, THSD1 variants showed reduced binding affinity to endogenous talin protein (comparing lane 3-7 to lane 2 in Fig. 4C). Further, we compared the effects of different THSD1 variants on mature adhesion number and cell attachment, and results showed that all of them have differentially reduced activity (Fig. 4D and Fig. 4E), supporting that compromised FA by THSD1 variants may contribute to IA pathogenesis.

\section{THSD1 is also present in endosomes}

THSD1 staining was also observed in discrete puncta that are not nascent FAs (Fig. 1). Since THSD1 is a single-span transmembrane protein and both its binding partners FAK and talin can localize in part in endosomes [8], we hypothesized that THSD1 may undergo endocytosis and thus localize at endocytic structures similar to many other single-span transmembrane proteins such as transferrin, or epidermal growth factor receptors (EGFRs). As shown in Fig. 5A, significant overlapping signals were detected between immunostained THSD1 with EEA1, an early endosome marker (See white arrows and overlapping signals in a4). Next, we checked two additional endosome markers CIMPR and Rab4 that are used for late and recycling endosomes, respectively. THSD1 localizes at these two different types of endosomes as judged by the overlapping signals (Fig. 5b4 and 5c4). Further, we also detected strong co-localization between THSD1 and LAMP1 (Fig. 5D), a marker for lysosomes, consistent with previous reports that one route of endocytosis is to send its target to lysosomes for degradation. All these data suggest that THSD1 can be redistributed by the endocytic network.

\section{Endosome-mediated THSD1 dynamics regulate FA}

Since THSD1 localizes at nascent but not mature adhesions, it is likely that THSD1 traffics to other places during such a transition, possibly by endosome-mediated redistribution. To test whether endosome-mediated redistribution affects THSD1 activities such as increasing mature adhesions number and cell attachment, we firstly treated HeLa cells expressing either control GFP or THSD1-GFP with hypertonic sucrose, a widely-used agent to block endocytosis [18]. Judged by the number of zyxin-positive puncta, the mature adhesions induced by THSD1 expression was significantly reduced upon sucrose treatment (comparing the fourth to the third bar in the left graph of Fig. 6A), while the basal level was minimally affected. In addition, cell attachment assay demonstrated that endocytosis inhibition compro- 
Fig. 6. Endosome-mediated THSD1 dynamics regulates focal adhesion. (A-C) HeLa cells expressing either 2ug control GFP or THSD1-GFP were treated by $0.4 \mathrm{M}$ hypertonic sucrose $(\mathrm{A})$, $100 \mu \mathrm{M}$ MDC (B) or $5 \mu \mathrm{g} / \mathrm{ml}$ filipin (C) for 1 hour before being subjected to immunostaining against zyxin (left graphs in A, B and $\mathrm{C}$ ) or cell attachment assay (right graphs in A, B and C). * = $\mathrm{p}<0.05$. (D) 2ug FLAG-THSD1 was transfected and expressed for 48 hours before being treated by MDC $(100 \mu \mathrm{M})$ or filipin (5 $\mu \mathrm{g} / \mathrm{ml}$ ) for 1 hour. Endogenous FAK was co-immunoprecipitated by anti-talin antibody. (E) A working model summarizing the role of THSD1 at the interface between FA and endosome dynamics.

mised THSD1-induced cell attachment (comparing the fourth to the third bar in the right panel of Fig. 6A). These data suggest that

A
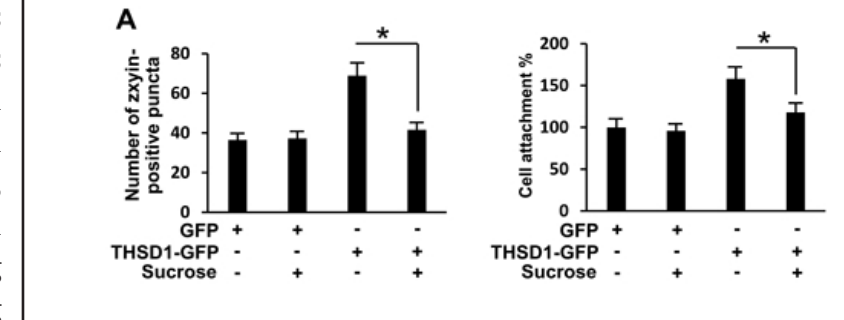

B
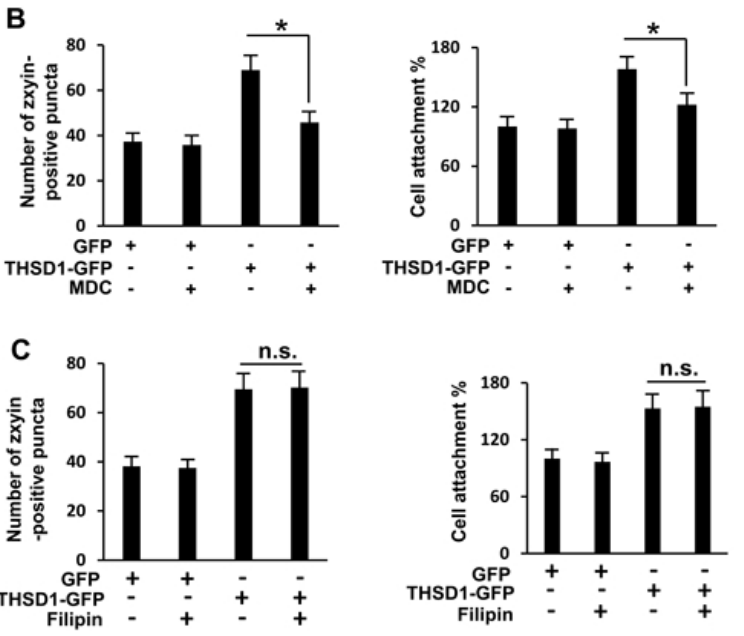

D
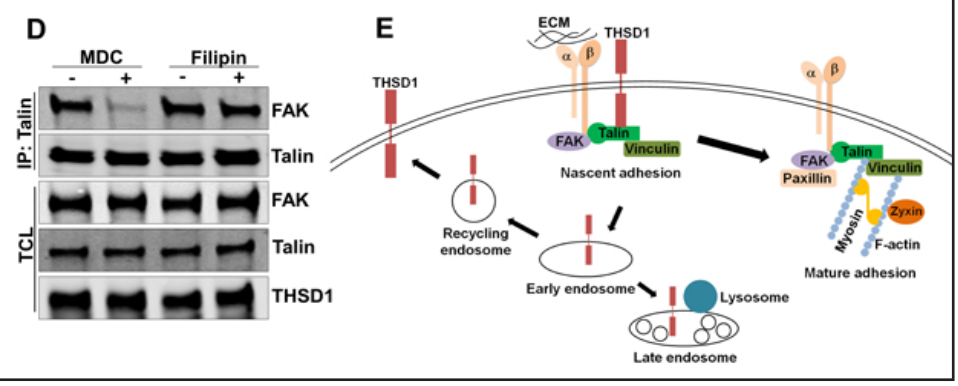
endocytosis is required for THSD1-induced FA number.

Although hypertonic sucrose is well known to block clathrin-mediated endocytosis, it also non-specifically affects caveolae-mediated endocytosis [18], which relies on the lipid raft formation and functions independently of clathrin. To further clarify which pathway is involved, we tested two other drugs including monodansylcadaverine (MDC) and filipin, which specifically inhibit the above two types of endocytosis respectively [18]. As shown in Fig. 6B, similar to hypertonic sucrose treatment, MDC inhibits THSD1-induced mature adhesions number and cell attachment (comparing the fourth to the third bar), while filipin treatment showed little effect (Fig. 6C), suggesting that clathrin-mediated but not caveolaemediated endocytosis regulates THSD1 activity.

Next, we examined whether disrupting the endosome-mediated THSD1 dynamics also dampens the nascent adhesions assembly, a prerequisite step for mature ones and cell attachment. As previously described, a critical event occurring during nascent adhesion assembly is the physical interaction between FAK and talin [6], which is enhanced in the presence of THSD1 (Fig. 4). We found that co-immunoprecipitated FAK in the presence of THSD1 was reduced upon MDC but not filipin treatment (comparing the second to the first lane, and the fourth to the third lane in Fig. 6D), consistent with above observations that clathrin-mediated but not caveolae-mediated endocytosis plays a critical role in regulating THSD1 normal functions.

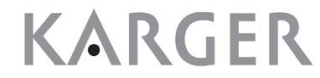




\section{Cellular Physiology Cell Physiol Biochem 2017;43:2200-2211

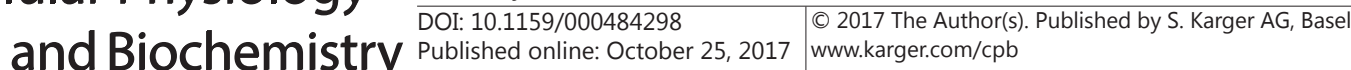 \\ Rui et al.: Endosome-Mediated THSD1 Dynamics Regulate FA}

\section{Model}

As summarized in Fig. 6E, THSD1 is a single-span transmembrane protein that localizes at nascent adhesions by physically interacting with FAK-talin-vinculin complex. However, when nascent adhesions mature and contain additional adaptor and structural proteins such as zyxin and actin filament, THSD1 leaves for other subcellular locations. One plausible route for THSD1 redistribution is through endocytosis, since it was observed located at different endocytic compartments including early, late, and recycling endosomes (see its dynamic localizations at the left and bottom area). Such endosome-mediated THSD1 dynamics play an important role in efficient nascent adhesion assembly possibly by providing more recycled THSD1 to new sites where nascent adhesions initiate.

\section{Discussion}

THSD1 is a nascent FA protein

Although many proteins have been identified for mature focal adhesion, the composition of nascent adhesion is largely unknown, especially in endothelial cells that express THSD1 [4]. Here, we provide compelling evidence to show that THSD1 is a novel nascent adhesion protein that physically forms a multimeric protein complex with FAK-talin-vinculin, which are three components of nascent adhesion (Fig. 1 and 2). Furthermore, the lack of co-localization between THSD1 and mature adhesion marker zyxin supports that THSD1 predominantly functions at earlier stage of FA assembly (Fig. 1B and Fig. 2B).

Interestingly, THSD1 promoted FAK-talin interaction but has no effect on talin-vinculin binding. FAK binds to the N-terminal FERM domain of talin [6], while THSD1 may bind to the adjacent area to facilitate FAK-talin interaction. Further domain mapping between THSD1 and talin will be necessary to functionally dissect the precise protein interaction region(s). It is worth noting that $\mathrm{C}$-terminal of talin constitutes a rod domain made of repetitive a-helix conformation where multiple vinculin binding sites exist [19] that may explain why the talin-vinculin binding affinity was not affected by THSD1 (Fig. 3D).

In addition to interacting with FAK, the N-terminal FERM domain of talin also binds to two other proteins including PIPK1 $\gamma$ and filamentous actin (F-actin) [19]. It was demonstrated that PIPK1 $\gamma$ increases the amount of PIP2 that can activate talin. More importantly, F-actin binding to talin provides a basis for generating the contractile force, which is critical for mature FA formation [3]. Therefore, it would be interesting to see whether these talin-interacting proteins can compete with THSD1, which ultimately leads to its disappearance during the transition from nascent to mature adhesions (Fig. 1B and Fig. 6E). Alternatively, THSD1 may intrinsically lose its binding affinity to talin due to its conformational changes, which are possibly contributed by posttranslational modifications such as protein phosphorylation. FAK may play such a role since a potential FAK-recognized site Y530 exists in the C-terminal region of THSD1, based on an established algorithm GPS 3.0 (Group-based Prediction System, version 3.0). This could raise an interesting scenario that although THSD1 promotes FAK-talin interaction, FAK may in turn phosphorylate THSD1 and provide a negative feedback that drives THSD1 out of nascent adhesions during their transition to mature ones.

It is worth of noting that THSD1 variants identified from brain aneurysm patients showed reduced binding affinity to talin and compromised ability to promote FAK-talin interaction (Fig. 4B and 4C), implying that aberrant nascent adhesion formation may be one of the factors contributing to the IA pathogenesis. These variants may intrinsically adopt different conformations that possess weaker binding to talin. Interestingly, the location of three substituted amino acids for the variants G600E, P639L and T653I are close to each other and may reflect a specific region of THSD1 to interact with talin. In addition, T653 in wild-type THSD1 is potentially phosphorylated upon the activation of vasopressin V2 receptor [20], a G protein-coupled receptor signaling pathway. Loss of such phosphorylation in THSD1 variant T653I may provide an alternative mechanism for how the normal function 


\section{Cellular Physiology Cell Physiol Biochem 2017;43:2200-2211

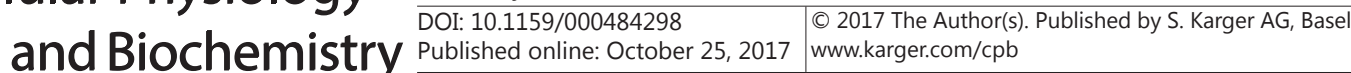 \\ Rui et al.: Endosome-Mediated THSD1 Dynamics Regulate FA}

of THSD1 is compromised during IA pathogenesis. In addition, it will be interesting to see whether there are any small non-coding RNAs regulating THSD1 stability and function, since some microRNAs were reported to play a role in intracranial aneurysm formation and rupture $[21,22]$.

\section{Potential role of THSD1 at the interface between FA and endosome}

THSD1 locates at nascent but not mature adhesions (Fig. 1), and meanwhile at endosomes of different stages (Fig. 5). It is likely that endosome-mediated THSD1 redistribution plays a role in its dichotomous FA localizations. In particular, the co-localization of THSD1 with recycling endosome labeled by Rab4 suggests that a redistribution pathway may help internalize THSD1 from previous nascent adhesion and deliver it to the next one, in order to increase the total amount of THSD1 that is accessible to nascent adhesions (Fig. 5C). Such endocytosis-mediated THSD1 dynamics positively regulates its activities, which was further confirmed by several following functional tests. For example, general inhibition of endocytosis by hypertonic sucrose abolishes THSD1-induced focal adhesion number and cell attachment (Fig. 6A). Intriguingly, a recent report also supports that endocytosis is able to positively regulate FA [8], in contrast to its traditional role in termination of integrin signals [7]. In particular, it was demonstrated that endocytosed integrin maintains active conformation when FAK localizes at the recycling endosome and interacts with talin and PIPK1 $\gamma$ to activate it [8]. It is possible that THSD1 may be co-endocytosed with integrin and facilitate integrin in active state since it can promote FAK-talin interaction, and the corecycled active integrin and THSD1 may initiate nascent adhesion in a more efficient way. Further investigations are required to clarify whether and how THSD1 utilizes the above plausible mechanisms including in quantity increasing the amount of accessible THSD1 to or in quality providing more THSD1-associated active integrin complex for nascent adhesions.

Clathrin-mediated endocytosis pathway is one of the best characterized among others. Indeed, its inhibition by MDC reduced the THSD1-induced FA number and cell attachment, possibly due to impaired FAK-talin interaction (Fig. 6D). In contrast, another clathrin-independent, caveolae-mediated endocytosis pathway shows little effect on THSD1 activities (Fig. 6C). These results suggest that clathrin-mediated but not caveolae-mediated redistribution of THSD1 is involved in its functional regulation. Nonetheless, gain-of-function approaches in our studies render the amount of potential ligand(s) for THSD1 insufficient and our experimental settings might only trigger clathrin-mediated endocytosis. Such a ligand dosage-induced switch has been shown to regulate EGFR internalization where a lower concentration of EGF ligand induces clathrin-mediated endocytosis while EGF in higher doses triggers caveolae-mediated endocytosis. Interestingly, clathrin-mediated endocytosis prefers its target receptor such as EGFR for recycling while caveolae-mediated endocytosis targets it for degradation [23]. Future investigations on the identification and characterization of the ligand(s) for THSD1 would help further dissect the role of endocytosis in THSD1 normal functions. Moreover, THSD1 ligand(s) may be a novel therapeutic target. In general, more ligands induce more frequent internalization of receptors, and such an accelerated endosome dynamics may compensate for the compromised nascent FA assembly due to THSD1 loss-of-function rare variants or even reduced level of expression, the latter of which has been reported in sporadic IA patients [24].

\section{Acknowledgements}

We appreciate the critical reading of this manuscript by Dr. Joanna O'Leary. The project was supported in part by R03NS087416 grant from the US National Institutes of Health.

\section{Disclosure Statement}

The authors have no conflicts to disclose. 


\section{Cellular Physiology Cell Physiol Biochem 2017;43:2200-2211

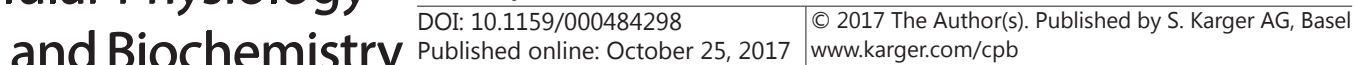

\section{References}

1 Santiago-Sim T, Depalma SR, Ju KL, McDonough B, Seidman CE, Seidman JG, Kim DH: Genomewide linkage in a large Caucasian family maps a new locus for intracranial aneurysms to chromosome 13q. Stroke 2009;40:S57-60.

-2 Santiago-Sim T, Fang X, Hennessy ML, Nalbach SV, DePalma SR, Lee MS, Greenway SC, McDonough B, Hergenroeder GW, Patek KJ, Colosimo SM, Qualmann KJ, Hagan JP, Milewicz DM, MacRae CA, Dymecki SM, Seidman CE, Seidman JG, Kim DH: THSD1 (Thrombospondin Type 1 Domain Containing Protein 1) Mutation in the Pathogenesis of Intracranial Aneurysm and Subarachnoid Hemorrhage. Stroke 2016;47:3005-3013.

-3 Sun Z, Guo SS, Fassler R: Integrin-mediated mechanotransduction. J Cell Biol 2016;215:445-456.

-4 Sun Z, Lambacher A, Fassler R: Nascent adhesions: from fluctuations to a hierarchical organization. Curr Biol 2014;24:R801-803.

5 Haining AW, Lieberthal TJ, Del Rio Hernandez A: Talin: a mechanosensitive molecule in health and disease. FASEB J 2016;30:2073-2085.

6 Lawson C, Lim ST, Uryu S, Chen XL, Calderwood DA, Schlaepfer DD: FAK promotes recruitment of talin to nascent adhesions to control cell motility. J Cell Biol 2012;196:223-232.

7 Ezratty EJ, Bertaux C, Marcantonio EE, Gundersen GG: Clathrin mediates integrin endocytosis for focal adhesion disassembly in migrating cells. J Cell Biol 2009;187:733-747.

-8 Nader GP, Ezratty EJ, Gundersen GG: FAK, talin and PIPKIgamma regulate endocytosed integrin activation to polarize focal adhesion assembly. Nat Cell Biol 2016;18:491-503.

-9 Xu Z, Rui YN, Balzeau J, Menezes MR, Niu A, Hagan JP, Kim DH: Highly efficient one-step scarless protein tagging by type IIS restriction endonuclease-mediated precision cloning. Biochem Biophys Res Commun 2017;490:8-16.

10 Rui YN, Xu Z, Chen Z, Zhang S: The GST-BHMT assay reveals a distinct mechanism underlying proteasome inhibition-induced macroautophagy in mammalian cells. Autophagy 2015;11:812-832.

-11 Rui YN, Xu Z, Patel B, Chen Z, Chen D, Tito A, David G, Sun Y, Stimming EF, Bellen HJ, Cuervo AM, Zhang S: Huntingtin functions as a scaffold for selective macroautophagy. Nat Cell Biol 2015;17:262-275.

12 David-Morrison G, Xu Z, Rui YN, Charng WL, Jaiswal M, Yamamoto S, Xiong B, Zhang K, Sandoval H, Duraine L, Zuo Z, Zhang S, Bellen HJ: WAC Regulates mTOR Activity by Acting as an Adaptor for the TTT and Pontin/ Reptin Complexes. Dev Cell 2016;36:139-151.

13 Rui Y, Xu Z, Lin S, Li Q Rui H, Luo W, Zhou HM, Cheung PY, Wu Z, Ye Z, Li P, Han J, Lin SC: Axin stimulates p53 functions by activation of HIPK2 kinase through multimeric complex formation. EMBO J 2004;23:45834594 .

14 Humphries MJ: Cell adhesion assays. Methods Mol Biol 2009;522:203-210.

15 de Hoog CL, Foster LJ, Mann M: RNA and RNA binding proteins participate in early stages of cell spreading through spreading initiation centers. Cell 2004;117:649-662.

16 Zaidel-Bar R, Ballestrem C, Kam Z, Geiger B: Early molecular events in the assembly of matrix adhesions at the leading edge of migrating cells. J Cell Sci 2003;116:4605-4613.

$>17$ Geiger B, Spatz JP, Bershadsky AD: Environmental sensing through focal adhesions. Nat Rev Mol Cell Biol 2009;10:21-33.

18 Ivanov AI: Pharmacological inhibition of endocytic pathways: is it specific enough to be useful? Methods Mol Biol 2008;440:15-33.

19 Critchley DR, Gingras AR: Talin at a glance. J Cell Sci 2008;121:1345-1347.

20 Rinschen MM, Yu MJ, Wang G, Boja ES, Hoffert JD, Pisitkun T, Knepper MA: Quantitative phosphoproteomic analysis reveals vasopressin V2-receptor-dependent signaling pathways in renal collecting duct cells. Proc Natl Acad Sci U S A 2010;107:3882-3887.

-21 Sun L, Zhao M, Zhang J, Lv M, Li Y, Yang X, Liu A, Wu Z: MiR-29b Downregulation Induces Phenotypic Modulation of Vascular Smooth Muscle Cells: Implication for Intracranial Aneurysm Formation and Progression to Rupture. Cell Physiol Biochem 2017;41:510-518.

22 Liu D, Han L, Wu X, Yang X, Zhang Q, Jiang F: Genome-wide microRNA changes in human intracranial aneurysms. BMC Neurol 2014;14:188.

23 Haglund K, Dikic I: The role of ubiquitylation in receptor endocytosis and endosomal sorting. J Cell Sci 2012;125:265-275.

-24 Pera J, Korostynski M, Krzyszkowski T, Czopek J, Slowik A, Dziedzic T, Piechota M, Stachura K, Moskala M, Przewlocki R, Szczudlik A: Gene expression profiles in human ruptured and unruptured intracranial aneurysms: what is the role of inflammation? Stroke 2010;41:224-231. 